\title{
Cure kinetic study of methacrylate-POSS copolymers for ocular lens
}

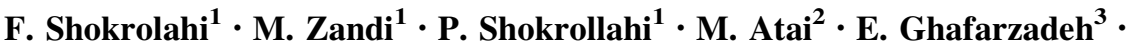 \\ M. Hanifeh ${ }^{1}$
}

Received: 8 July 2017/ Accepted: 5 October 2017/Published online: 25 October 2017

(c) The Author(s) 2017. This article is an open access publication

\begin{abstract}
The physical, mechanical and biological properties of multicomponent acrylate-based hard lenses are directly influenced by degree of conversion achieved during copolymerization. In this research, polyhedral oligomeric silsesquioxane (POSS) acrylate is introduced into the polymer backbone in combination with hydroxyethyl methacrylate, dimethyl itaconate, methyl methacrylate, 3-(trimethoxysilyl) propyl methacrylate and triethylene glycol dimethacrylate in free-radical bulk polymerization. Kinetics of curing process was investigated by two techniques: differential scanning calorimetry and FTIR spectroscopy. Reaction kinetics in free-radical bulk polymerization of the system was studied by isothermal DSC performed at $65,75,85$, and $95^{\circ} \mathrm{C}$ using different quantities of initiator. Three compounds were prepared in different concentrations $(0.2,0.4$ and $0.6 \mathrm{~mol} \%)$ of $2,2^{\prime}$ azobisisobutyronitrile as initiator. Conversion rate was calculated as a function of time using data obtained from DSC measurements. The kinetic parameters of the reaction such as reaction constants, reaction orders and activation energies were obtained from the isothermal DSC data according to the autocatalytic model developed by Kamal and Sourour. The results showed that the experimental
\end{abstract}

M. Zandi

m.zandi@ippi.ac.ir

1 Biomaterials Department, Iran Polymer and Petrochemical Institute, Pazhoohesh Blvd, Tehran-Karaj Hwy, 1497713115 Tehran, Iran

2 Science Department, Iran Polymer and Petrochemical Institute, Pazhoohesh Blvd, Tehran-Karaj Hwy, 1497713115 Tehran, Iran

3 Lassonde School of Engineering, York University, 4700 Keele Street, 11 Arboretum Ln, ON M3J 1P3 Toronto, Canada values were in good agreement with theoretically estimated values and our results may suggest that the polymerization reaction of this system is well described by Kamal's model. Cytotoxicity results, performed on extracts 28 days after PBS incubation, showed no toxicity of the materials extracted from the lenses indicating that they can be considered as safe materials in ocular lens applications. The viability and proliferation of L929 fibroblast cells in extracting media were followed by 3-[4,5-dimethylthiazol2-yl]-2,5-diphenyltetrazolium bromide (MTT) and they may have a great potential as ideal supporting lens in people who suffer from keratoconus disease.

Keywords Acrylate-POSS · Polyhedral oligomeric silsesquioxane $\cdot$ Kinetic study $\cdot$ Copolymerization $\cdot$ Contact lens

\section{Introduction}

There are many ophthalmic devices, especially designed for eye disorders to provide the best possible vision. Ocular lenses which are considered as medical devices can be worn to correct vision, cosmetic or therapeutic purposes. There are three types of lenses: rigid, soft and hybrid (semi-rigid) lenses due to selected materials and also final applications (Mannis et al. 2003).

Semi-rigid gas-permeable lenses are the primary candidates for correcting the impaired vision arising from deformed cornea. In one therapy methodology offered by Boston (USA), over-sized hard contact lenses of appropriate geometry are made of highly gas-permeable hard plastics that allow oxygen in reach the cornea. The original gas-permeable hard plastic lenses are mainly made of silicone acrylate-based monomers and supplied as a clear 
dome-shape, approximately the size of a nickel. The device fits under the eyelid overlaying the damaged cornea while leaving a space from the eye that is filled with sterile saline to provide a lubricating pool of artificial tear to the eye. Therefore, the key element of such treatments is the initial hard plastic from which the final device is made according to the patient's eye characteristics (Rathi et al. 2011). To fabricate biocompatible materials for ocular lenses, it is vital to consider polymerization reaction as well as the starting materials. In this research, a multicomponent acrylate-based copolymer especially designed in ocular lens application is developed.

One of the most interesting techniques for lens preparation is bulk polymerization of multi-functional acrylicbased monomers, because a variety of polymerization methods and systems are achievable. Free-radical bulk polymerization of vinyl monomers, characterized by autoacceleration, has been widely investigated (Peinado et al. 2002; Wang and Hutchinson 2010). Putting efforts on such investigations is rational because of fabricating the biocompatible materials, is vital to deeply understand and take control over the polymerization steps. The magnitude of such a realization and familiarity is more palpable when considering that the synthesized polymer is being utilized in manufacturing of biomedical devices. Biocompatibility and physical/mechanical properties of the products are greatly influenced by the approach taken to control the reaction. Heat of reaction is also directly linked with the reaction rate (Lee and Lee 1994). It is noticeable that freeradical polymerization of acrylic monomers is highly exothermic; when the reaction temperature is not controlled, it results in undesirable physical and mechanical properties (Vinnik and Roznyatovsky 2004; Woo and Seferis 1990). On the other hand, the presence of any residual unreacted monomer(s) causes some problems for the exposed cells, namely cytotoxicity in contact with the equipment. Hence, in order to obtain a safe product, it is very crucial to determine the exact time of reaction completion (Vermette et al. 2001).

Monomers used in this study generally contain active acrylic functionalities for free-radical polymerization which are performed in bulk. In this research, methacrylate polyhedral oligomeric silsesquioxane (POSS-acrylate) was introduced and according to our literature review, there are very few reports on the influence of POSS content on the polymerization kinetics of such systems.

Polyhedral oligomeric silsesquioxanes have attracted attention in nanocomposite technology because of inorganic silicate base with organic exterior. The introduction of POSS cages into copolymer backbone results in increased glass transition, decomposition temperature, and oxygen permeability as well as enhanced mechanical properties (Ghasaban et al. 2011; Ke et al. 2012). In our previous study, we also demonstrated an inspiring approach in the synthesis of biomaterials containing POSS cage directly joined to the acrylic-based polymer backbone to improve hardness without sacrificing other essential lens materials properties (Hanifeh et al. 2017). The focus of this present paper is placed on predicting the cure behavior and kinetics of curing reaction of the systems containing multicomponent acrylate-based monomers (hydroxyethyl methacrylate (HEMA), dimethyl itaconate (DMI), methyl methacrylate (MMA), 3-(trimethoxysilyl) propyl methacrylate (TMSPMA) and triethylene glycol dimethacrylate (TGDMA)). The effect of POSS-acrylate introduced into the polymer backbone in free-radical bulk polymerization was studied. Many techniques such as Fourier transform infrared spectroscopy (FTIR), Raman, electron paramagnetic resonance; nuclear magnetic resonance, differential scanning calorimetry (DSC) and differential thermal analysis (DTA) have been used to determine the degree of conversion. Among these, FTIR is the most frequently used method.

Many studies have also focused on the kinetic characterization of thermoset resin systems using DSC (Lam 1989; Ivankovic et al. 2006; Sbirrazzuoli and Vyazovkin 2002; Kamal 1974).

Calorimetry is a widely used method for measuring the reaction rate constants as well as orders of reactions. Here, we report on kinetics study of a multicomponent acrylatebased system, in which a combined curing agents system consisting of triethylene glycol dimethacrylate and POSS was used to render the composition a higher degree of biocompatibility. The reaction rate constants are measured at different temperatures as a function of initiator concentration and the data are compared with those calculated based on Kamal's model (Kamal 1974).

The results of this research provide insights into our future plan to design suitable lens for treatment of keratoconus which is the most prevalent primary corneal ectasia while the changes in ocular signs and symptoms depend on severity of the disease (Kennedy et al. 1986; Weed et al. 2008; Romero-Jiménez et al. 2010). The disease progress leads to significant loss of vision that cannot be compensated with spectacles. Patients with keratoconus have better vision by semi-rigid contact lenses since these lenses are placed in front of the cornea and pass the light rays to the retina.

\section{Experiments}

\section{Materials}

Hydroxyethyl methacrylate and methyl methacrylate were purchased from Merck, Germany. Triethylene glycol 
dimethacrylate, dimethyl itaconate, 2,2'-azobisisobutyronitrile (AIBN) and 3-(trimethoxysilyl) propylmethacrylate, were supplied by Sigma-Aldrich, Germany. AIBN was recrystallized in methanol prior to synthesis in order to remove any impurities. Methacrylate polyhedral oligomeric silsesquioxane (POSS-acrylate) was obtained from Hybrid Plastics, USA and used as received. 3-[4,5Dimethylthiazol-2-yl]-2,5 diphenyltetrazolium bromide MTT, alizarin red staining, dexamethasone and ascorbic acid (Sigma) were purchased from Sigma, UK, Roswell Park Memorial Institute-1640.

\section{Methods}

\section{Polymerization reaction}

The synthesis of silicon acrylate lens materials in combination with POSS-acrylate was the objective of this study. The polymerization of a number of carefully selected monomers was carried out taking a free-radical bulk polymerization approach for which the kinetics data as well as degree of conversion is reported here. Therefore, hydroxyethyl methacrylate, methyl methacrylate, triethylene glycol dimethacrylate, dimethyl itaconate, 3-(trimethoxysilyl) propylmethacrylate, and POSS-acrylate mixture were carefully mixed under $\mathrm{N}_{2}$ atmosphere until a homogenous mixture was obtained. Then, AIBN was added and the mixture was transferred into a proper mold. In the first step, polymer compounds were heated at optimized temperature $\left(65^{\circ} \mathrm{C}\right)$ for $24 \mathrm{~h}$. The lenses were removed from the mold and maintained at $45{ }^{\circ} \mathrm{C}$ for $48 \mathrm{~h}$ to complete the polymerization. In Fig. 1 , the polymerization reaction is shown schematically.

\section{Kinetic study}

To study the reaction kinetics as a function of initiator concentration, three formulations were prepared with different concentrations of initiator. The exact composition of each formulation is listed in Table 1.

As it is illustrated in Table 1, the concentration of all the constituents was kept constant for three different formulations, except AIBN, which was used at 3 different concentrations of $0.2,0.4$ and $0.6 \mathrm{~mol} \%$.

Dynamic DSC experiments were carried out on a Maia 200-F3, Netszch, Germany, at a heating rate of $10{ }^{\circ} \mathrm{C} / \mathrm{min}$ from ambient temperature until completion of the process in order to determine the total heat of fusion of cure reaction. The exothermic reaction was completed when the heat flow curve versus temperature was leveled off.

Isothermal DSC experiments were also performed on a Maia 200-F3, Netszch, Germany, at four different temperatures of $65,75,85$ and $95{ }^{\circ} \mathrm{C}$ for each formulation.

\section{FTIR study}

FTIR analysis was carried out to identify the functional groups and degree of polymerization. For this purpose, samples were ground to powder, then mixed with potassium bromide and pressed into discs. To determine the optimal time and temperature in order to complete the polymerization reaction, FTIR spectroscopy was also used.

\section{Light absorption test}

To measure the transparency of the transmitted light absorption test, a $0.1 \mathrm{~mm}$ thick lens was cut. Calibration was performed to measure the amount of visible light passing through a black sheet as a zero-percent reference, and the unobstructed environment between the bulb and the light-absorbing sensor as one hundred-percent reference.

\section{Cytotoxicity Evaluation}

The cytotoxicity of fabricated lenses was evaluated by extraction method through which the samples were immersed into PBS solution at $37{ }^{\circ} \mathrm{C}$ for 28 days. Then, the extraction was applied to the testing system containing L929 fibroblast cells for $24 \mathrm{~h}$ and cytotoxicity was studied in accordance with ISO 10993.

The viability of L929 fibroblast cells was also studied using 3-[4,5-dimethylthiazol-2-yl]-2,5-diphenyltetrazolium bromide (MTT). A batch of $100 \mu \mathrm{L}$ MTT solution with 900 $\mu \mathrm{L}$ medium was incubated with the cells in the wells at $37{ }^{\circ} \mathrm{C}$ for $3 \mathrm{~h}(n=3$ for each measurement day). After $3 \mathrm{~h}$, dimethyl sulfoxide solution (DMSO, Sigma Chemical Germany) was added to dissolve formazan crystals. The absorbance values of formazan solutions, obtained from the above given substrates, were measured using an ELIZA reader at $570 \mathrm{~nm}$ (Bio-Tek ELx800). All calculations were made using SPSS statistical software. Data are presented as mean $\pm \mathrm{SD}$, recorded in triplicate.

\section{Results and discussion}

\section{Kinetic study}

This study also focused on the kinetics of free-radical polymerization of a multicomponent (meth) acrylate system containing variable amounts of thermal initiator. The especial emphasis in this study was focused on the development of a comprehensive kinetics model for free-radical polymerization at initiation stage. Here, kinetic parameters such as activation energy, pre-exponential factor and order of reaction are calculated based on the data obtained from differential scanning calorimetry (DSC), measurements in 


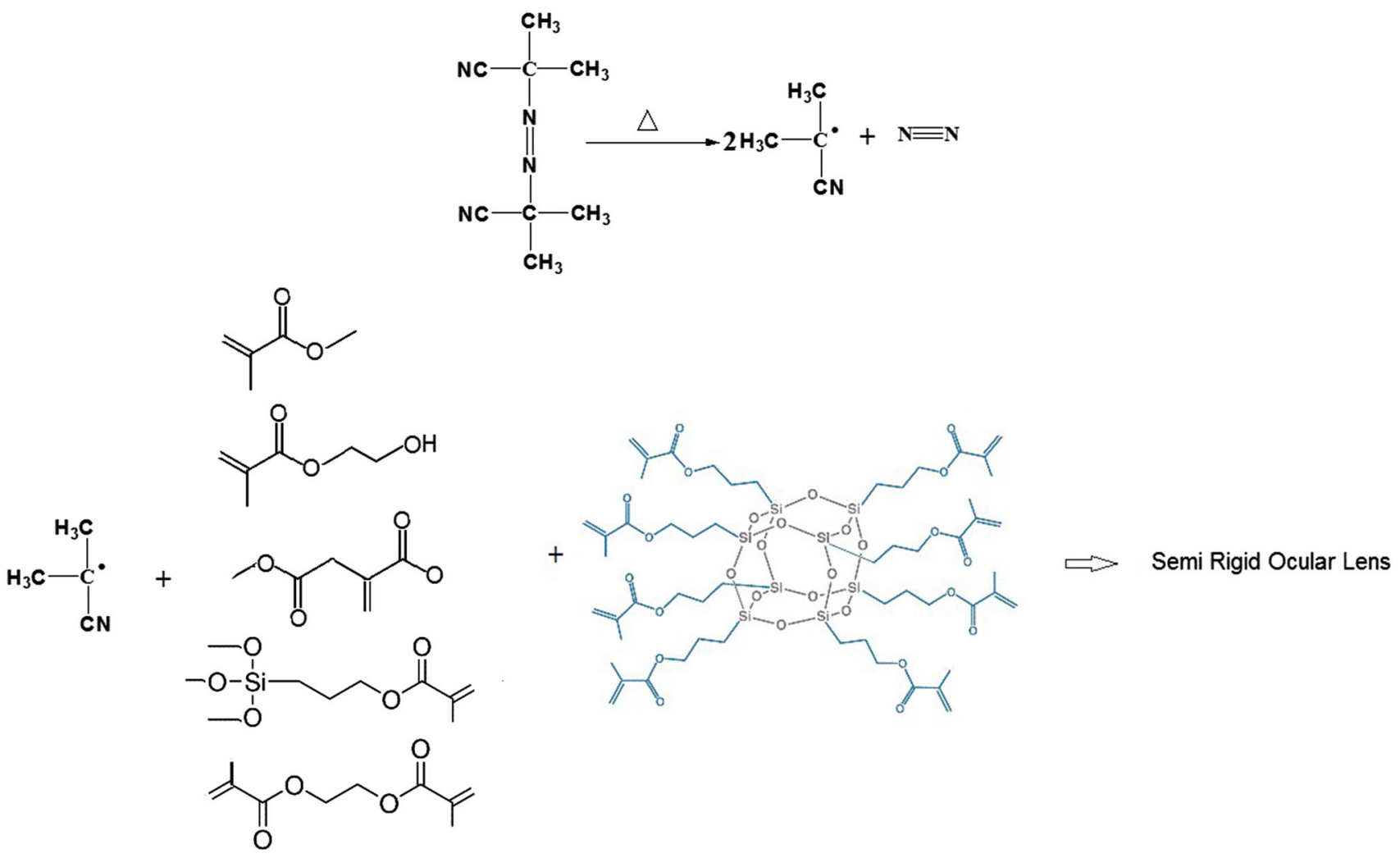

Fig. 1 Schematic presentation of polymerization reaction

Table 1 Chemical composition of the samples used for kinetics study. The relative concentration of the constituents was kept constant except AIBN, which was used at three different concentrations

\begin{tabular}{llllllll}
\hline & AIBN $(\mathrm{g})$ & TEGDMA $(\mathrm{g})$ & HEMA $(\mathrm{g})$ & POSS $(\mathrm{g})$ & DMI $(\mathrm{g})$ & DMS $(\mathrm{g})$ & NMA $(\mathrm{g})$ \\
\hline 1 & 0.16 & 0.2 & 0.06 & 0.06 & 0.3 & 0.4 & 0.24 \\
2 & 0.32 & 0.2 & 0.06 & 0.06 & 0.3 & 0.4 & 0.24 \\
3 & 0.72 & 0.2 & 0.06 & 0.06 & 0.3 & 0.4 & 0.24 \\
\hline
\end{tabular}

a set of isothermal experiments especially designed and performed for the sake of kinetics study. When the curing reaction is the only thermal event in the reaction, then the reaction rate or conversion rate must equal the rate of heat flow $(d Q / d t)$ provided by differential scanning calorimeter.

The values obtained in DSC experiments are used to measure kinetic parameters of the reaction using the equations given in this article. For every isothermal experiment, two lines are drawn from starting and ending points of exothermic peak to the baseline. The area under the curve (AUC), obtained from all the area bounded by the curve, is calculated to measure the heat of reaction. The graphs are divided into smaller parts and the reaction rate, heat of reaction and the heat of the remaining reaction are recorded for each time interval. A fraction is obtained for every area. For kinetic studies by means of DSC, the rate of reaction is supposed to be proportional to the rate of heat generation and can be expressed using Eq. (1) (Kamal 1974; Xu et al. 2017).

$\frac{d \alpha}{\mathrm{dt}}=\frac{\frac{d H}{\mathrm{dt}}}{\Delta \mathrm{H}_{t o t}}$

where $\frac{d \alpha}{\mathrm{dt}}$ is the rate of the reaction and $\Delta \mathrm{H}_{\text {tot }}$ is the total heat of the reaction. The total heat of reaction generated to reach full conversion, $\Delta \mathrm{H}_{\text {tot }}$, was calculated by DSC dynamic scan at $10{ }^{\circ} \mathrm{C} / \mathrm{min}$ for each composition. In Fig. 2, the A series, the conversion rate versus time is illustrated for different amounts of initiator at four different temperatures of $65,75,85$ and $95{ }^{\circ} \mathrm{C}$. Two-phase autocatalytic equation of Kamal was used to calculate the reaction rate constants. Equation 2 shows Kamal's model (Kamal 1974; Prime 1981; Kenny 1994).

$\frac{d \alpha}{d t}=\left(k_{1}+k_{2} \alpha^{m}\right)(1-\alpha)^{n}$ 

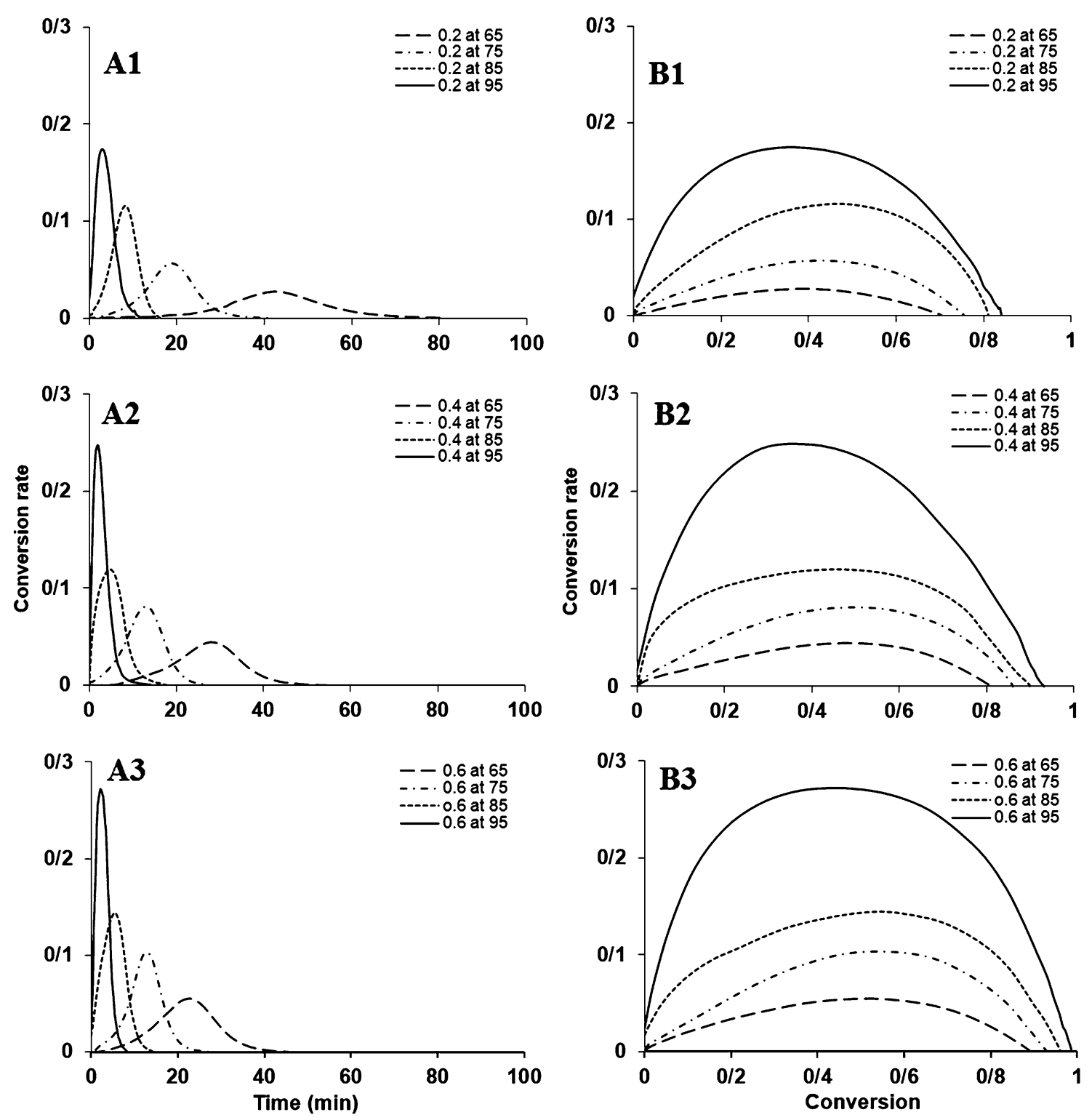

Fig. 2 Dependence of the conversion rate of polymeric systems curing time at different isothermal temperatures (the A series) and isothermal reaction rate of the polymeric systems curing as a function of conversion at different isothermal temperatures (the $\mathrm{B}$ series)

where $n$ and $m$ are the parameters of the order of reaction; $k_{1}, k_{2}$ are the reaction rate constants at temperature $T$. The conversion rate is calculated with respect to the time by Eqs. 3 and 4, using the heat flow data obtained from DSC measurements (Khanna and Chanda 1993).

As illustrated in Fig. 2, the B series, the conversion rate is dependent on the reaction temperature and as the polymerization temperature increases, the peak of the conversion rate rises higher and its maximum point moves towards shorter times.

This figure shows the values for the conversion rate with respect to the conversion percentage for different amounts of initiator at $65,75,85,95{ }^{\circ} \mathrm{C}$.
The results reveal that the maximum rate of reaction at 65,75 and $85{ }^{\circ} \mathrm{C}$ has occurred at $50 \%$ conversion, while at $95{ }^{\circ} \mathrm{C}$ the maximum reaction rate has been registered at about $35-40 \%$ conversion.

Reaction rate constants are also temperature dependent and they are calculated by Arrhenius equation (Eq. 3):

$k_{i}(T)=A e^{\frac{-E}{R T}} \quad i=1,2$

where $A$ is the pre-exponential factor, $E$ is the activation energy, $T$ is the absolute temperature, and $R$ is the universal gas constant. To calculate the unknown values of Eq. 4 in our reaction, this equation is rewritten as a logarithm (Eq. 4) 
$\ln \left[\frac{d \alpha}{d t}\right]=\ln \left(k_{2} \alpha^{m}+k_{1}\right)+n \ln [1-\alpha]$

when Eq. 4 is rewritten, it can be assumed as Eq. 5 or Eq. 6

$\ln \left[\frac{d \alpha}{d t}\right]-\ln \left(k_{2} \alpha^{m}+k_{1}\right)=n \ln [1-\alpha]$

$\ln \left\{\left[\left(\frac{d \alpha}{d t}\right) /(1-\alpha)^{n}\right]-k_{1}\right\}=\ln k_{2}+m \ln \alpha$

The value $\alpha$ at time 0 , on $\alpha(\mathrm{t})$ curve plotted versus $\ln \frac{d \alpha}{d t}$, gives $\mathrm{K}_{1}$. Graphical representation of Eq. 6 has been illustrated in Fig. 3. The slope of the initial linear part of the curve in Fig. 3 provides the first estimation for $n$.

Next, the estimated values of $k_{1}$ and $n$ using Eq. (4) are introduced into Eq. 6 by $\ln \left[\left(\frac{d \alpha}{d t}\right) /(1-\alpha)^{n}\right]-k_{1}$ plotted versus $\ln \alpha$. The slope of the initial linear part of this curve gives $\mathrm{m}$, while the $y$-intercept is $\ln k_{2}$ (Fig. 4). Having $m, k_{1}$ and $k_{2}$ values in hand, $\mathrm{n}$ is re-calculated from Eq. (5). The $n$ obtained this way is different from the initial estimation and all calculations are repeated using the second value estimated for $n$. This continues until the difference between two values is less than one percent. Next, $\ln \left[\left(\frac{d \alpha}{d t}\right) /\left(k_{2} \alpha^{m}-k_{1}\right)\right]$ was plotted against $\ln (1-\alpha)$ in order to recalculate $n$ (Fig. 5).

The reaction rate constant dependence on temperature is given by an Arrhenius type of equation as is shown in Eqs. (1) and (2). The reaction rate constant is calculated using Eq. (7), then

$k_{1}(T)=A e^{\frac{-E}{R T}}$

where $A$ is a pre-exponential factor, $E$ is the activation energy, $T$ is the absolute temperature, and $R$ is the universal

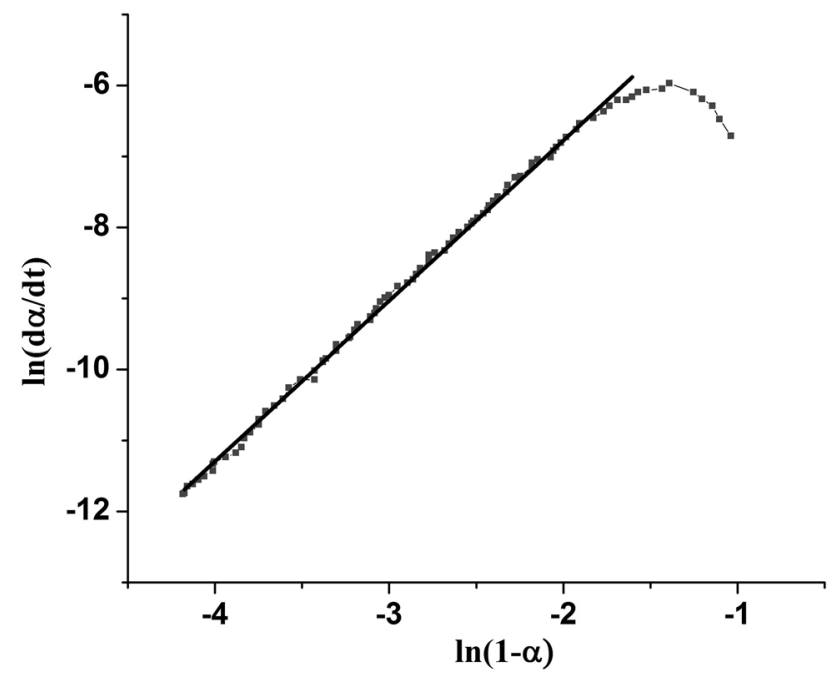

Fig. 3 Variation of $\ln \frac{d \alpha}{d t}$ with $\ln (1-\alpha)$ Slope of the initial linear area of the curve gives an initial estimation for $\mathrm{n}$

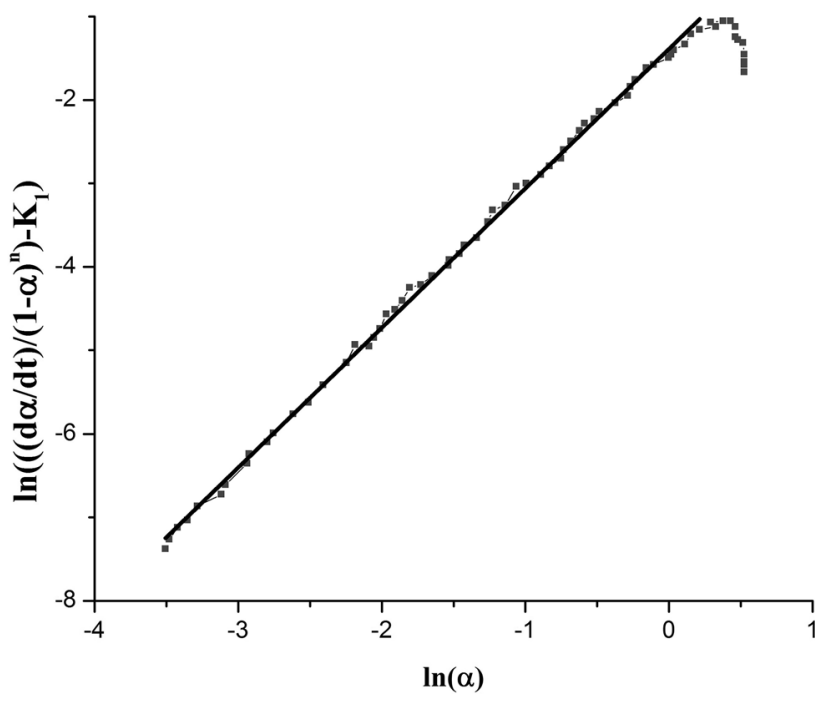

Fig. 4 Curve of $\ln \left[\left(\frac{d \alpha}{d t}\right) /(1-\alpha)^{n}\right]-k_{1}$ versus $\ln \alpha$. From this curve $m$ (slope of the linear part), and $\ln \alpha$ ( $y$-intercept), are easily calculated

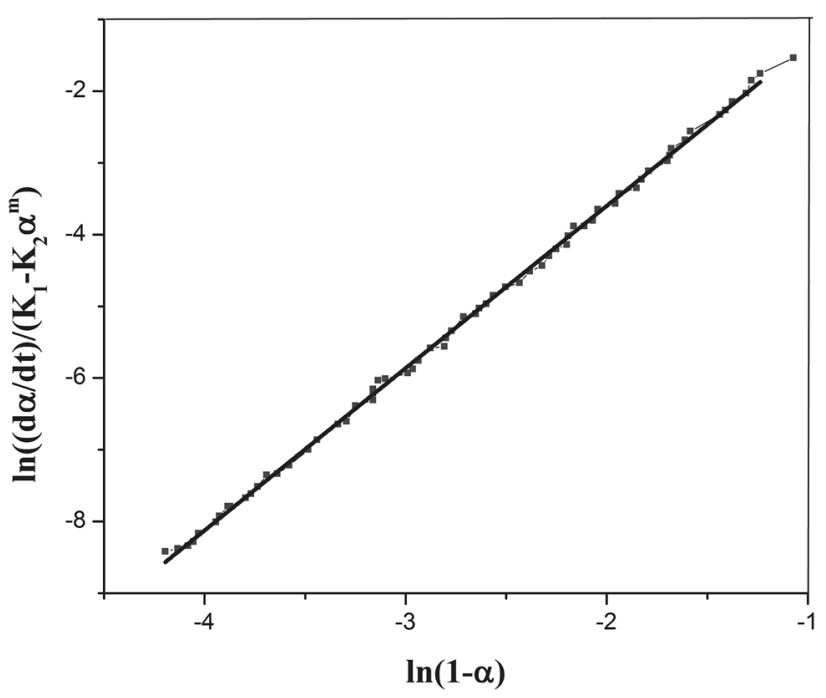

Fig. $5 \ln \left[\left(\frac{d \alpha}{d t}\right) /\left(k_{2} \alpha^{m}-k_{1}\right)\right]$ variation with $\ln (1-\alpha)$ obtained from Eq. 4 and Eq. 5 for recalculating

gas constant. Equation (7) is then rewritten as a logarithm (Eq. 8).

$\ln [k(T)]=\ln [A]-\frac{E}{R T}$

If Eq. 8 is plotted as $\ln [k(T)]$ versus $\frac{1}{T}$, this relation indicates a first degree equation with $\frac{-E}{R}$ gradient and $\ln [\mathrm{A}]$ as intercept. The values obtained for reactions rate constants are listed in Table 2 for different concentrations of the initiator.

A comparison between the experimental values obtained from DSC data and the theoretical values estimated based on Kamal's model is illustrated in Fig. 6. As it can be observed in this figure, there is a good conformity between 
Table 2 Values calcualted for the kinetic parameters of the reaction. $\mathrm{n}$ and $\mathrm{m}$ are mean values

\begin{tabular}{lllll}
\hline Initiator $(\mathrm{Mol} \%)$ & Reaction rate constant & Temperature dependence & $m$ & $n$ \\
\hline 0.2 & $\mathrm{~K}_{1}$ & 0 & 0.92 & 1.12 \\
& $\mathrm{~K}_{2}$ & $4.50 \times 10^{6} \exp (-6093 / \mathrm{T})$ & & \\
0.4 & $\mathrm{~K}_{1}$ & $2.99 \times 10^{6} \exp (-8355 / \mathrm{T})$ & 0.86 & 1.12 \\
& $\mathrm{~K}_{2}$ & $5.78 \times 10^{7} \exp (-7475 / \mathrm{T})$ & & \\
0.6 & $\mathrm{~K}_{1}$ & $2.49 \times 10^{5} \exp (-5446 / \mathrm{T})$ & 0.82 & 1.12 \\
& $\mathrm{~K}_{2}$ & $7.75 \times 10^{6} \exp (-5798 / \mathrm{T})$ & & \\
\hline
\end{tabular}

the estimated values and the experimental values measured here (Hanifeh et al. 2015).

The results in Table 2 show the kinetic rate constants increased with catalyst concentration at the same curing temperature. Meanwhile, the values of each reaction order, $\mathrm{m}$ and $\mathrm{n}$, are slightly decreased. Comparisons between the experimental and theoretical cure parameters based on the autocatalytic model show good agreements up to about $80 \%$ conversion (where the reaction is kinetically controlled), but beyond that region modest deviations are observed.

\section{Degree of conversion}

The degree of conversion (DC) is determined by measuring the intensity (height or area of absorption band) of unreacted aliphatic $\mathrm{C}=\mathrm{C}$ double bonds at $1638 \mathrm{~cm}^{-1}$ relative to the amount of $\mathrm{C}=\mathrm{O}$ bonds at $1720 \mathrm{~cm}^{-1}$ whose intensity is unaltered during polymerization process $[24,11]$. In this technique, it is unnecessary to take into account the sample thickness. Therefore, the percentage of aliphatic $\mathrm{C}=\mathrm{C}$ bonds remaining unreacted during the polymerization is calculated by the following equation:

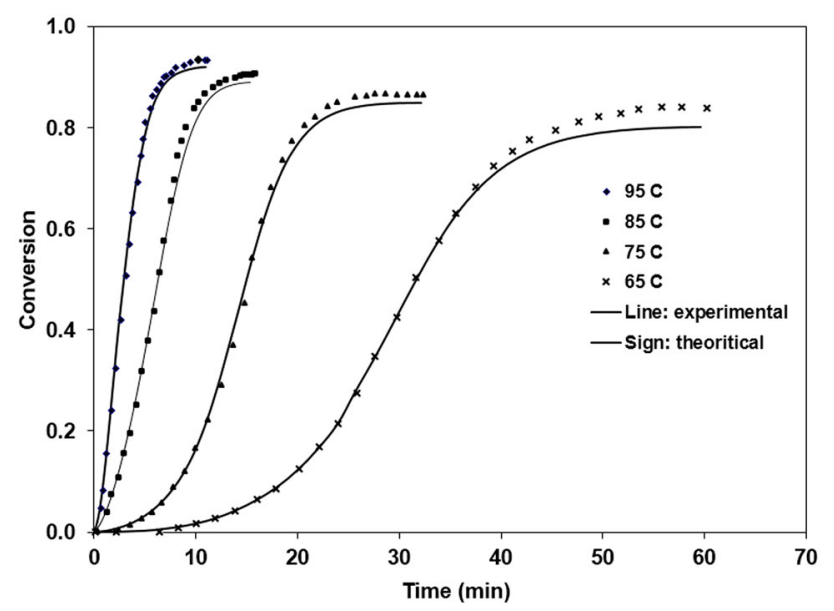

Fig. 6 Comparison of experimental conversion data for the polymeric system with the theoretical values calculated based on Kamal's Model, as a function of time at different isothermal curing temperatures

$$
\begin{aligned}
(\% \mathrm{DC})= & 1-[[\mathrm{Abs}(\mathrm{C}=\mathrm{C} \text { double bonds }) \\
& / \mathrm{Abs}(\mathrm{C}=\mathrm{O} \text { bonds })] \text { polymer : } \\
& {[\mathrm{Abs}(\mathrm{C}=\mathrm{C} \text { double bonds }) / \mathrm{Abs}(\mathrm{C}=\mathrm{O} \text { bonds })] \times 100 }
\end{aligned}
$$

To determine the appropriate reaction temperature and time, the reaction was carried out at temperatures of 65,75 , 85 and $95{ }^{\circ} \mathrm{C}$ for $24 \mathrm{~h}$ on the sample as mentioned in Table 3. The prepared samples were taken at each individual temperature of infrared range and the reaction progress was examined.

In Fig. 7, infrared spectra obtained from synthesized copolymers at different temperatures are shown.

DC calculated at different temperatures from the FTIR spectra is tabulated in Table 4.

The degree of conversion calculation shows that by increasing the temperature, the DC increases which could be due to rapid formation of free radicals, increasing the number of effective monomer collisions and increasing the number of monomers with sufficient energy for reaction.

\section{Light absorption test}

To evaluate the transparency of the lenses, the light absorption test has been carried out. The light passage rate for this sample is more than $92 \%$ the same as light passage rate for pure methyl methacrylate $(92 \%)$. Therefore, the manufactured specimen has a suitable light passage rate for use as a transparent material.

\section{Cytotoxicity test}

L929 fibroblast cell culturing was used to confirm that fabricated lenses have no toxicity impact on the fibroblast cells. Therefore, lenses synthesized in different temperatures were defined for culturing the fibroblast cells. The cells were cultured in the extract from lenses synthesized at 50, 60 and $70{ }^{\circ} \mathrm{C}$. As it is illustrated in Fig. 8, the cells grow and proliferate into the extract and it shows no toxic substance into the extraction media (Fig. 9).

MTT assay which is sensitive to detect small differences in cell number was used on the lens materials to analyze fibroblast cells growth rate. With regard to fibroblast cells 
Table 3 Degree of conversion of samples synthesized as given in Table 2

\begin{tabular}{lllllll}
\hline AIBN $(\mathrm{g})$ & TEGDMA $(\mathrm{g})$ & HEMA $(\mathrm{g})$ & POSS $(\mathrm{g})$ & DMI $(\mathrm{g})$ & TMSPMA $(\mathrm{g})$ & MMA $(\mathrm{g})$ \\
\hline 0.328 & 0.312 & 0.25 & 0.5 & 1.5 & 2.5 & 1.8 \\
\hline
\end{tabular}

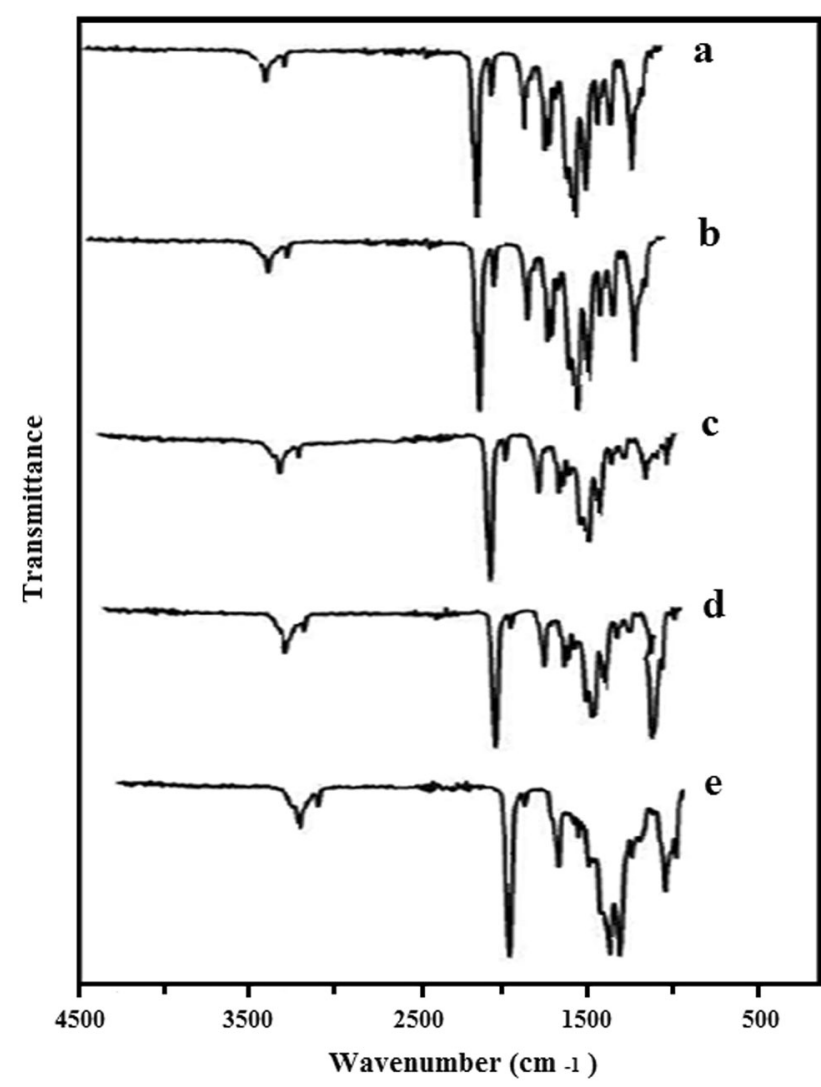

Fig. 7 Infrared spectra obtained from synthesized copolymers: a before heating and at different temperature b 65, c 75, d 85 and e $9{ }^{\circ} \mathrm{C}$ for $24 \mathrm{~h}$

Table 4 Values calculated for kinetic parameters of the reaction. $n$ and $m$ are mean values

\begin{tabular}{lllll}
\hline Reaction temperature $\left({ }^{\circ} \mathrm{C}\right)$ & 65 & 75 & 85 & 95 \\
Degree of conversion DC $(\%)$ & 55 & 69 & 84 & 96 \\
\hline
\end{tabular}

behavior in vicinity of the lenses, the results demonstrate some minor changes in cell number. According to these results, lenses synthesized at $65-85{ }^{\circ} \mathrm{C}$ show less toxicity.

\section{Conclusion}

For synthesis of silicone acrylate-based lens materials containing POSS, a free-radical bulk polymerization was chosen. In this study, curing reaction kinetics was investigated by means of two techniques: differential scanning calorimetry and FTIR spectroscopy. Kinetics parameters of the polymerization systems specially designed for contact

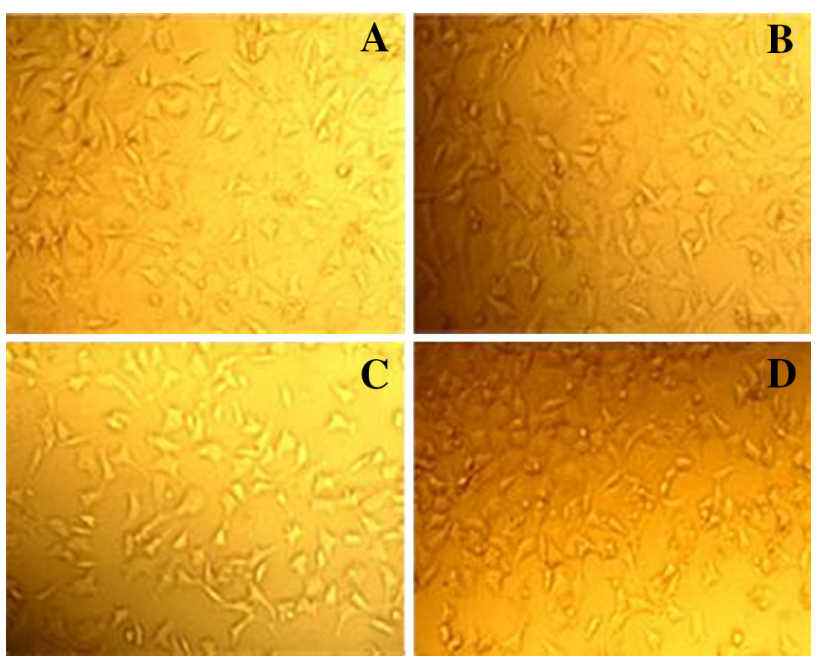

Fig. 8 Light microscope images of L929 fibroblast cell culture in extracts from contact lenses. a control b synthesized at $50{ }^{\circ} \mathrm{C}$, c synthesized at $60{ }^{\circ} \mathrm{C}$ and $\mathbf{d}$ synthesized at $70^{\circ} \mathrm{C}$ for $24 \mathrm{~h}$

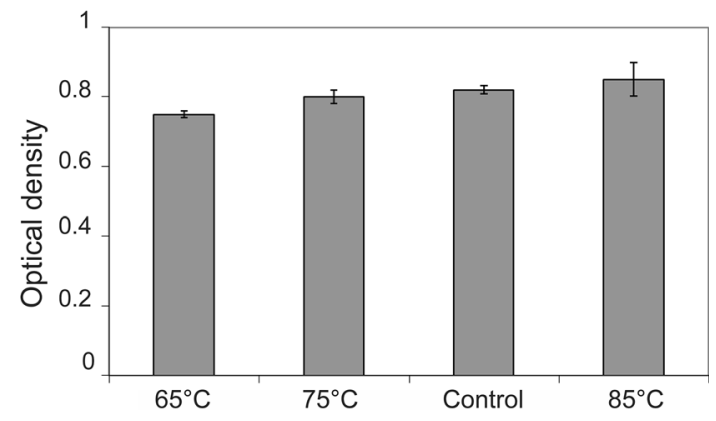

Fig. 9 Cell viability versus synthesizing temperature: a cell viability of L929 fibroblast cells tested at $50{ }^{\circ} \mathrm{C}, 60{ }^{\circ} \mathrm{C}$ and $70{ }^{\circ} \mathrm{C}$ in compared to tissue culture plate as control from MTT assay. Data are expressed as mean $\pm \mathrm{SD}(n=3)$. Data are subject to Dunnet one-way analysis of variance (ANOVA), $* * * p<0.001$

lens formulations were estimated based on Kamal's autocatalytic model. The results from DSC experiments revealed that the reaction rate increased with temperature for all copolymers and that peak reaction rate in reaction rate versus time curve moved towards shorter times. Moreover, the maximum rate of reaction for the reactions at temperatures 65,75 and $85{ }^{\circ} \mathrm{C}$ has occurred at conversion levels of 70-40\%, while the maximum rate of reaction at $95{ }^{\circ} \mathrm{C}$ occurred at about $35 \%$ conversion. These results clearly showed that as the curing temperature was increased, the system exhibited better agreement with the Kamal's autocatalytic model and the best agreement was observed for the system cured at $95{ }^{\circ} \mathrm{C}$. 
The completion in reaction of the multicomponent systems containing POSS was studied with FTIR spectroscopy, for which the results showed a perfect match with the results of DSC experiments (complete removal of the $\mathrm{C}=\mathrm{C}$ vibration at $1638 \mathrm{~cm}^{-1}$ in the FTIR spectrum at $\left.95{ }^{\circ} \mathrm{C}\right)$.

Cytotoxicity tests showed that the growth and proliferation of cells within the extracted media are comparable with the cells growth and proliferation in the culture medium (as control).

Open Access This article is distributed under the terms of the Creative Commons Attribution 4.0 International License (http://crea tivecommons.org/licenses/by/4.0/), which permits unrestricted use, distribution, and reproduction in any medium, provided you give appropriate credit to the original author(s) and the source, provide a link to the Creative Commons license, and indicate if changes were made.

\section{References}

Ghasaban S, Atai M, Imani M, Zandi M, Shokrgozar MA (2011) Photo-crosslinkable cyanoacrylate bioadhesive: shrinkage kinetics, dynamic mechanical properties, and biocompatibility of adhesives containing TMPTMA and POSS nanostructures as crosslinking agents. J Biomed Mater Res Part A 99A:240-248

Hanifeh M, Zandi M, Shokrollahi P, Ataei M, Ghafarzadeh E, Shokrolahi F (2015) Fabrication of multicomponent silicone acrylate based copolymer utilized in ocular lenses. International Conference and exhibition on advanced and nano materials

Hanifeh M, Zandi M, Shokrollahi P, Ataie M, Ghafarzadeh E, Askari F (2017) Compositional design and taguchi optimization of hardness properties in silicone based ocular lenses. Prog Biomater. doi:10.1007/s40204-017-0065-y

Ivankovic M, Brnardic I, Ivankovic H, Mencer HJ (2006) DSC study of the cure kinetics during nanocomposite formation: epoxy/poly (oxypropylene) diamine/organically modified montmorillonite system. J Appl Polym Sci 99(2):550-557

Kamal MR (1974) Thermoset characterization for moldability analysis. Polym Eng Sci 14:231-239

Ke F, Zhang C, Guang S, Xu H (2012) POSS Core star-shape molecular hybrid materials: effect of the chain length and POSS content on dielectric properties. J Appl Polym Sci 127:2628-2634

Kennedy RH, Bourne WM, Dyer JA (1986) A 48-year clinical and epidemiologic study of Keratoconus. Am J Ophthalmol 101(3):267-273

Kenny JM (1994) Determination of autocatalytic kinetic model parameters describing thermoset cure. J Appl Polym Sci 51:761-764

Khanna U, Chanda M (1993) Kinetics of anhydride curing of isophthalic diglycidyl ester using differential scanning calorimetry. J Appl Polym Sci 49(2):319-329

Lam PWK (1989) The characterization of thermoset cure behavior by differential scanning calorimetry. Part II: effects of low-profile additives. Polymer Compos 10(6):439-448

Lee JH, Lee JW (1994) Kinetic parameters estimation for cure reaction of epoxy based vinyl ester resin. Polym. Engin. Sci. $34: 742-749$
Mannis MJ, Zadnik K, Coral-Ghanem C, Kara-José N (2003) Contact lenses in ophthalmic practice. Springer Science \& Business Media, Berlin

Peinado C, Salvador AF, Basega J, Catalina F (2002) Following in situ photoinitiated polymerization of multifunctional acrylic monomers by fluorescence and photocalorimetry simultaneously. Polymer 43(19):5355-5361

Prime RB (1981) Thermal characteristics of polymeric materials. In E Turi (Ed) Academic Press, New York

Rathi VM, Mandathara PS, Dumpati S (2011) Boston ocular surface prosthesis: an Indian experience. Ind J Ophth 59:279-281

Romero-Jiménez M, Santodomingo-Rubido J, Wolffsohn JS (2010) Keratoconus: a review. Contact Lens Anterior Eye 33(4):157-166

Sbirrazzuoli N, Vyazovkin S (2002) Learning about epoxy cure mechanisms from isoconversional analysis of DSC data. Thermochim Acta 388(1):289-298

Vermette P, Griesser HJ, Laroche G, Guidoin R (2001) Biomedical application of polyurthane. Tissue Eng Intell 6:2-8

Vinnik M, Roznyatovsky VA (2004) Kinetic method by using calorimetry to mechanism of epoxy-amine cure reaction. J Therm Anal Cal 76:285-293

Wang W, Hutchinson RA (2010) Free-radical acrylic polymerization kinetics at elevated temperatures. Chem Eng Technol 33(11):1745-1753

Weed KH, MacEwen CJ, Giles T, Low J, McGhee CN (2008) The dundee University Scottish Keratoconus study: demographics, corneal signs, associated diseases, and eye rubbing. Eye 22(4):534-541

Woo EM, Seferis JC (1990) Cure kinetics of epoxy/anhydride thermosetting matrix systems. J Appl Polym Sci 40:1237-1256

Xu X, Zhou Q, Song N, Ni Q, Ni L (2017) Kinetic analysis of isothermal curing of unsaturated polyester resin catalyzed with tert-butyl peroxybenzoate and cobalt octoate by differential scanning calorimetry. DOI, J Therm Anal Calorim. doi:10.1007/ s10973-017-6246-z

F. Shokrolahi received her B.Sc. from Iran University of Science and Technology and M.Sc. from Amir Kabir University of Technology. She joined Iran Polymer and Petrochemical Institute (IPPI) as a researcher then, where she received her Ph.D. in 2011. She is an assistant Prof. at Department of Biomaterials at IPPI now and the main topics of her research are design of Polyurethanes/Polyurethane ureas and nano-composites for biomedical applications and Tissue engineering.

M. Zandi received the B.Sc. Degree in chemistry from Shahid Beheshti University and M.Sc. Degree in Polymer Chemistry from Sharif University of Technology, Tehran, Iran, in 1990 and 1996 respectively. She has got a Ph.D. in polymer chemistry from Duisburg-Essen University, Germany, in 2008. She started working at Iran Polymer and Petrochemical Institute (IPPI) as a research scientist. She is now an associate professor at IPPI and her research interests include polymeric biomaterials and tissue engineering.

P. Shokrollahi received her B.Sc. in Chemistry from Tehran University and M.Sc. in Analytical Chemistry from Shahid Beheshti University, Tehran, Iran. She joined Iran Polymer and Petrochemical Institute (IPPI) as a researcher. She went to University of Cambridge and received her Ph.D. in Polymer chemistry in 2009. She is an Assistant Prof. at Department of Biomaterials at IPPI and main topics 
of her research are design of supramolecular polymers for biomedical applications and Tissue engineering.

M. Atai received the B.Sc. and M.Sc. Degrees from Amir Kabir University of Technology, Tehran, Iran in 1991 and 1994. He started working at Iran Polymer and Petrochemical Institute (IPPI) as researcher and received his Ph.D. in 2004 from IPPI. He is now professor at IPPI and his research interests include polymeric biomedical and dental materials.
E. Ghafarzadeh received his Ph.D. from Ecole Polytechnique de Montreal (Canada, 2008) in Electrical Engineering and completed an NSERC Postdoctoral Fellowship at University of California, Berkeley, in Bioengineering (2010-2012). He is now an Assistant Professor of Electrical Engineering and Computer Science in York University, Toronto, Canada.

M. Hanifeh received his M.Sc. Degree in Polymer technology from Iran Polymer and Petrochemical Institute in 2012 